LPHE-MS-11-02/ CPM-11-02

\title{
The Yang Monopole in IIA Superstring: Multi-charge Disease and Enhançon Cure
}

\author{
Adil Belhaj ${ }^{1,2,5 *}$, Pablo Diaz ${ }^{3 \dagger}$, Antonio Segui ${ }^{4 \ddagger}$ \\ ${ }^{1}$ Lab Phys Hautes Energies, Modelisation et Simulation, Faculté des Sciences, Rabat, Morocco \\ ${ }^{2}$ Centre of Physics and Mathematics, CPM-CNESTEN, Rabat, Morocco \\ ${ }^{3}$ National Institute for Theoretical Physics, University of Witwatersrand, South Africa \\ ${ }^{4}$ Departamento de Fisica Teorica, Universidad de Zaragoza, 50009-Zaragoza, Spain \\ ${ }^{5}$ Groupement National de Physique des Hautes Energies, Siège focal: FSR, Rabat, Morocco
}

November 3, 2018

\begin{abstract}
A brane picture in Type IIA superstring for the Yang Monopole is reconsidered. It makes use of D2 and D4-branes wrapped on cycles in the K3 surface. When the model was first presented some problems concerning the charges of the monopoles arised. In this paper, they are shown to be cured by the model itself. Surprisingly, the incompatibility between the multi-charge configuration and the spherical symmetry of the Yang Monopole is seen in the brane description as the emergence of the enhançon shell and the fuzzy geometry. This consistency is deep and surprising, and is the point that triggered this work. It nontrivially relates a purely geometrical problem in ordinary spacetime with the emergence of noncommutative geometries. Besides, this paper includes an extended model for $S O$ (4)-monopoles, a T-dual model in Type IIB superstring and an analysis on the possible duality between our model and another setup in M-Theory/Heterotics for the Yang monopole found before.
\end{abstract}

Keywords: Yang Monopole, Supertring theory, Dualities, M-theory, K3 surface.

*belhaj@unizar.es

†pablo.diazbenito@wits.ac.za

${ }_{\ddagger}^{\ddagger}$ segui@unizar.es 


\section{Contents}

1 Introduction

2 Type IIA superstring construction of the Yang monopole 5

2.1 Superstringy construction of the Yang monopole . . . . . . . . . . . 5

2.2 Relation with the heterotic M-theory Yang monopole configuration . . 7

2.3 Six dimensional effective field theory . . . . . . . . . . . . . 9

2.4 Dual model in Type IIB superstring . . . . . . . . . . . . . . . . . 9

$3 S O(4)$-monopoles $\quad 10$

4 The multi-charge disease and the enhançon cure $\quad 12$

4.1 The problem ......................... 12

4.2 The enhançon . . . . . . . . . . . . . . . . 13

4.3 A way out of spherical symmetry . . . . . . . . . . . 16

5 Connection with fuzzy spheres

6 Discussion and open questions $\quad 20$ 


\section{Introduction}

Based on the idea that string theories at low energies must be well approached by supergravity, we have been searching for a brane model in type IIA string theory of the Yang monopole [1]. In the brane picture shown in [1] most of the features of the Yang construction where successfully reproduced, namely the charges, the nonabelian field and the point-like behavior of Yang configurations. Some corners of the brane model remained unanswered, however, questions whose investigation have revealed some unexpected deep connections between gauge theory and brane physics and have finally lead to this paper.

Before going deeper into this let us recall some general details of the Yang monopole.

As a pure gauge solution of the Yang monopole [2] was first constructed as a generalization of the Dirac monopole [3]. The Yang configuration is characterized by the flux of the four-form field $\operatorname{Tr} F \wedge F$, charged under the $S U(2)$ gauge group, across the four dimensional sphere that covers the origin in a $5+1$ dimensional space-time. It corresponds to the conformal mapping into $S^{4}$ of the BPST Euclidean instanton solution [4]. Again, the origin is singular but now the energy of this solitonic configuration is well behaved in the UV regime, although IR divergences linearly appear. The total energy inside a four sphere is proportional to its radius. In this realization, the flux is quantized but now, the magnetic charge of the Yang monopole can take only two values $\{+1,-1\}$ [2]. This charge, which may correspond to the self-dual and anti-selfdual BPST instanton configurations respectively, is given by the integral over $S^{4}$ of the second Chern class $\operatorname{Tr}(F \wedge F) / 8 \pi^{2}$. The Yang monopole can be easily generalized to higher even dimensional space-time where the configuration is characterized by $2 n$-form $F^{n}$. Explicit solutions can be systematically obtained in [5, 6, 7, 8].

We would like to stress at this point the well known fact that the Yang monopole, due to its spherical symmetry, can carry just two charges [2]. This is a purely gauge requirement in the sense that, unlike the fuzzy backgrounds which emerge in the brane picture, the charge analysis from the gauge theory point of view concerns topologically nontrivial configurations of gauge fields in an ordinary 4-dimensional sphere. Indeed, in the process of generalizing the Yang construction for larger symmetry groups and dimensions, there is a systematic way of finding the number of configurations (charges) that an arbitrary gauge field with symmetry group $G$ on a $K / H$ symmetric (ordinary) base space can show once $K$-symmetry on the field configuration is imposed [9]. The configurations over a symmetric space are labeled by the so-called Wang maps. Wang maps are homomorphisms between $H$ (the isotropy group) and the symmetry group of the gauge field $G$ up to isomorphism. Note, again, that the 
whole analysis in the general case in [9] involves an ordinary spacetime as a base space. Note, also, that when we talk about spherical symmetry we refer to the case in which the symmetric base space is a sphere. For the Yang case, the base space is $S^{4} \subset R^{5,1}$, the $K$-symmetry is $S O(5) 1$, and the gauge group $G$ is $S U(2)$.

In the brane model presented in [1] the two charge-nature of the Yang monopole seemed to hold nicely as associated with the two ways a single D4-brane can wrap a 2-cycle of the K3 surface. Specifically, the construction given in [1] needs D2 and D4-branes on the $\mathrm{K} 3$ surface. In this brane picture, the $S U(2)$ gauge group of the Yang construction is engineered by means of a D2-brane wrapping shrinking 2-cycles inside the K3 surface. The Yang monopole comes up when a D4-brane wraps the whole K3 and dimensional reduction to 6-spacetime dimensions is performed. In this way, the Yang properties above mentioned are encoded in the K3 surface features. However, a careful look reveals a mechanism that allows us to obtain a multi-charge spherically symmetric configuration. Basically, the mechanism consists of adding subsequent D4-branes to the setup at no cost of energy since the branes are taken to be BPS states. The addition of $N$ D4-branes would increase the charge in $N$ units without loosing spherical symmetry since the branes are point-like in $5+1$ dimensions. It would lead, as said, to a multi-charge spherically symmetric configuration.

At this stage, the brane setup would be ruined as a model for the Yang monopole given that, as said before, no spherical SU(2) configuration over $S^{4}$ can be multicharged. However, a deeper investigation on the brane physics reveals the appearance of an enhançon shell [10, 11, 12, 13, 14, 15, 16, 17] and a fuzzy geometry at the core of the monopole for the multi-charge case. Fuzzy geometry explicitly breaks spherical symmetry. So the two-charge property of the spherically symmetric gauge theory configuration gets restored. The surprise comes because spherical symmetry is broken in the brane picture by a non-ordinary spacetime background: the (noncommutative) fuzzy 4-sphere that the enhançon mechanism brings aside. In this highly nontrivial way, which involves fuzzy geometries, a gauge requirement for the two allowed charges of the Yang configuration is recovered in the brane picture.

Apart from the discussion of the multicharge configurations and the broken spherical symmetry, this paper presents a new brane model for $S O(4)$-monopoles in six dimensions. The construction is inspired in the model presented in [1]. It is, say, its natural extension. The number of charges labeling the topologically different brane configurations in this case is 4 . Needles to say that the same argument used for the Yang monopole holds for the $S O(4)$ construction and also prevents it from having an

\footnotetext{
${ }^{1}$ The symmetry is actually spin(5), the double cover of $S O(5)$ as proved in [9], but this subtlety is not going to make much difference in our discussion.
} 
infinite tower of charges without breaking spherical symmetry.

The organization of this paper is as follows. In section 2 we will review the model proposed in [1], discuss the relation with the M-theory/Heterotic string setup and find a useful T-dual version of the Type IIA model in Type IIB superstring. In section 3 we will extend the logic of the model for the case of $S O(4)$-monopoles. Section 4 deals with the core of the discussion. It is devoted to the discussion of the main objections concerning the multiple charge configurations the model presented at first sight. There will be offered, to these objections, a solution coming from the model itself by a purely stringy effect: the enhançon mechanism and the fuzzy geometry which comes along with it. Both, intriguing phenomena, which are better visualized in the T-dual model. Finally, a brief conclusion sums up the main points of the paper and brings some open questions.

\section{Type IIA superstring construction of the Yang monopole}

The idea of finding a brane picture for Yang monopoles is not new though. Before [1], it was suggested the possibility of considering the Yang Monopole in M-theory [18]. In this regard, a heterotic M-theory realization was soon proposed [19]2. In particular, [19] shows that M5-brane may have boundaries on M9-branes, where the boundary is a D4-brane with an infinite tension so its centre of mass is not free to move. The boundaries may be identified with Yang monopoles. Indeed, in this heterotic Mtheory picture there are two Yang monopoles which correspond to the ends of the oriented M5-brane which stretches between two M9-branes. Each monopole (each end) is charged under an $S U(2)$ subgroup of $E_{8}$ with the topological charges $\{+1,-1\}$ respectively. Using string/string duality and the result of [19], we give a string realization of the Yang monopole for a six dimensional Type IIA superstring obtained from the compactification on a local description of the $K 3$ surface in the presence of wrapped D-branes. Then we study its relation with the M-theory model.

\subsection{Superstringy construction of the Yang monopole}

Consider a local description of the K3 surface where the manifold develops a su(2) singularity (known as $A_{1}$ singularity). This singularity corresponds to a vanishing two-sphere. Near such a singular point, the K3 surface can be identified with the asymptotically locally Euclidean (ALE) complex space which is algebraically given

\footnotetext{
${ }^{2}$ Independently, a matrix model of the Yang monopole was given in [20].
} 
by

$$
f(x, y, z)=x y-z^{2}=0,
$$

which is singular at $x=y=z=0$. In two-dimensional $N=2$ linear sigma model with only on $3 U(1)$ gauge symmetry, the resolution of this singularity is related to the D-term described by the following bosonic potential $V\left(\phi_{1}, \phi_{2}, \phi_{3}\right)$

$$
\left.V\left(\phi_{1}, \phi_{2}, \phi_{3}\right)=\left(\left|\phi_{1}\right|^{2}-2\left|\phi_{2}\right|^{2}+\left|\phi_{3}\right|^{2}\right)-R\right)^{2} \text {, }
$$

where $R$ is the $U(1)$ Fayet-Iliopoulos (FI) parametert [21]. Geometrically, this corresponds to replace the singular point $x=y=z=0$ by the 2-sphere $S^{2}$ defined by $V=\phi_{2}=0$, which is the only non-trivial 2-cycle on which we can wrap D2-branes. In order to geometrically engineer the $\mathrm{SU}(2)$ gauge symmetry only the compact piece containing the $S^{2}$ is necessary [22]. Now, the system consists of Type IIA D2-branes wrapping around $S^{2}$. This gives a pair of massive vectors $W^{ \pm}$, one for each of the two possible ways of the wrapping. The masses of these particles are proportional to the volume of the 2-sphere. They are charged under the $U(1)$ gauge field obtained by decomposing the type IIA three-form in terms of the harmonic 2-form on the 2-sphere and the 1-form gauge field in six dimensional space-time. In the limit where the 2sphere shrinks, the $W^{ \pm}$particles become massless and, together with the one form gauge field, generate the $S U(2)$ adjoint representation. This will be identified with the gauge symmetry of our Yang monopole. We have obtained the electrically charged sector, associated to D2-branes wrapping two vanishing cycles in the K3 surface. Lifting consistently to 11 dimensions, the M2-brane is encountered. The magnetic Yang monopoles can be identified with D4-branes, totally wrapped on the K3 surface. As consequence, they generate the magnetic objects in the six dimensional space-time. This is expected from the fact that the D4-brane is the only magnetic object in Type IIA superstring theory which can be obtained from the M5-brane and gives a pointlike particle after wrapping the $\mathrm{K} 3$ surface. Owing to the spherical symmetry of the six dimensional configuration and on the fact that, as seen above, the gauge group origin is linked to the singular limit of the geometry, we strongly believe that all Yang monopole properties should be derived from the K3 surface data.

Schematically, the ten dimensional spacetime where Type IIA lives is occupied as follows. If we consider that the K3 surface extents along dimensions 6789, and the vanishing 2-cycle of $\mathrm{K} 3$ at 67 positions, then the D2-brane is at 067 and the D4-brane at 06789 .

\footnotetext{
${ }^{3}$ For the ALE geometry $A_{n}$ the gauge group is $U(1)^{n}$.

${ }^{4}$ In this way, one sees that the $U(1)$ Cartan subgroup of the $S U(2)$ symmetry of the singularity of $K 3$ carries the gauge symmetry of the $N=2$ supersymmetric linear sigma model.
} 
We will show that the charges $\{+1,-1\}$ can have different compatible K3 surface interpretations. First, the different ways in which D4-branes are wrapped on the K3 surface. They are classified by its fourth homotopy group. As seen before, in order to construct the $S U(2)$ gauge group, it is necessary to work with a local model of K3 with a singularity $A_{1}$. The deformed geometry is given by the product of the complex $\mathbf{C}$ plane and a two sphere $S^{2}$. Since $\Pi_{q}(X \times Y)=\Pi_{q}(X) \times \Pi_{q}(Y)$, we have the following remarkable relation

$$
\pi_{4}\left(A_{1}\right) \sim \pi_{4}\left(S^{2}\right)=Z_{2} .
$$

The two charges of the Yang monopole are related to the two ways the geometry allows a D4-brane to wrap on it 5 .

It is known that the energy of the Yang monopole diverges linearly in spacetime. This fact is not manifest in this geometric construction. However, the divergence in the energy can be intuitively seen in the T-dual model (see sec. 2.4), where the D4brane turns into an infinite effective D1-brane which pulls at a pair of coincidents NS5-branes.

\subsection{Relation with the heterotic M-theory Yang monopole configura- tion}

The authors of [19] have suggested a Yang monopole representation with two $S U(2)$ gauge factors obtained by breaking the $E_{8} \times E_{8}$ heterotic gauge symmetry in ten dimensions. This breaking, $E_{8} \rightarrow E_{7} \times S U(2)$, could be related with the fact that the extremes of the M5-branes are located on the M9-branes and they are just the core of the Yang monopole. On the Type IIA side however there is only one $S U(2)$ factor which comes from a D2-brane wrapped around the collapsing $S^{2}$ inside the K3 surface. Lifting to M-theory the nature of this difference is appreciated. Reduction from 11 to 6 dimensions with sixteen supercharges can be performed in two dual ways depending on the action of the $Z_{2}$ symmetry on the five dimensional internal space $S^{1} \times T^{4}$. In the heterotic realization of M-theory, the $Z_{2}$ symmetry acts on the $S^{1}$ factor giving rise the segment between the two M9-branes, while in the Type IIA, M-theory, the symmetry acts on the $T^{4}$ factor producing the $K 3$ geometry. However, since these two M-theory compactifications are dual in six dimensions, the two above string Yang

\footnotetext{
${ }^{5}$ This last statement has left some room for controversy. After reading our paper [1], David Tong suggested that there should be five charges corresponding to the two ways the D4-brane and the D4antibrane wrap the $A_{1}$ manifold plus the trivial one (zero charge). We believe that David's idea is right for the case of extended-Yang monopoles, the ones with gauge group $S O(4)$, we will go on this point in subsection 3 .
} 
monopole realizations should be connected. In what follows, we will speculate on this link. Indeed, the K3 surface has two possible constructions as the target space of a sigma model. They depend on the R-symmetry of the supercharges. Previously, we have mainly concerned with $N=2$ sigma model, where the R-symmetry is supported by a $U(1)$ group, and the $K 3$ target space gets manifested as a Khäler manifold. Now, let us use the other realization of $K 3$ where the manifold is hyperkhaler and the corresponding sigma model involves eight supercharges and has a $S U(2)$ R-symmetry. Then, the $\{+1,-1\}$ charges of the Yang monopole can be explained by physical arguments when the $\mathrm{K} 3$ surface is constructed in terms of $N=4$ sigma model [23, 24]. This is related to the heterotic M-theory configuration where the Yang monopole has two copies in the boundaries of the M5-brane suspended between two M9-branes. Theses copies, with charges +1 and -1 , might be understood as two hypermultiplets appearing in the hyper-Khäler quotient construction of the $A_{1}$ space.

The six dimensional $S U(2)$ Yang Mills theory can also be obtained from the K3 surface that is realized in terms of $N=4$ supersymmetric sigma model. This sigma model has only one $U(1)$ gauge group, two hypermultiplets with charges $\left(q_{1}, q_{2}\right)$, and one isotriplet FI coupling $\vec{\xi}=\left(\xi_{1}, \xi_{2}, \xi_{3}\right)$ [23, 24]. The sigma model gauge symmetry is related to the Cartan subgroup of the six dimensional gauge group. In this construction, the K3 surface is expressed by the vanishing condition of the following D-terms

$$
\sum_{i=1}^{2} q_{i}\left(\phi_{i}^{\alpha} \bar{\phi}_{i \beta}+\phi_{i \beta} \bar{\phi}_{i}^{\alpha}\right)-\vec{\xi} \vec{\sigma}_{\beta}^{\alpha}=0 .
$$

The double index $(i, \alpha)$ of the scalars refers to the component field doublets $(\alpha)$ of the two hypermultiplets $(i)$, and $\sigma$ are the traceless $2 \times 2$ Pauli matrices. The condition under which the gauge theory flows in the infrared to $2 \mathrm{~d} N=4$ superconformal field theory, which is also the condition to have a hyper-Khäler Calabi-Yau background, is

$$
q_{1}+q_{2}=0 .
$$

This equation has different solutions that can be seen as redefinitions of the coupling constant $\vec{\xi}$. Due to its conformal invariance, the theory does not get affected by redefinitions of $\vec{\xi}$, so the charge can be fixed to -1 and +1 .

Let us discuss the construction of the $K 3$ surface in this case. The starting point consists of two hypermultiplets with four scalars each. They can be expressed as $\mathbf{R}^{4} \times \mathbf{R}^{4}$. The gauge invariance of each hypermultiplet (with +1 and -1 charge) under the $U(1)$ symmetry, together with the invariance under the $S U(2)$ R-symmetry that rotates the supercharges, enables us to express the $K 3$ surface locally as the following homogenous space

$$
\frac{\mathbf{R}^{4} \times \mathbf{R}^{4}}{U(1) \times S U(2)} .
$$


There is a $Z_{2}$ symmetry that interchanges the two hypermultiplets (the two $\mathbf{R}^{4}$ factors). We interpret the two $\mathbf{R}^{4}$ factors with their corresponding charges as the two Yang monopole copies which are the boundaries of the M5-branes on the two M9branes. At this stage, we can ask the following question, which is the role of the $S U(2)$ R-symmetry in this construction? The answer of this question lies on the association of the R-symmetry with the instantonic nature of the M5-brane in the context of the heterotic M-theory picture.

\subsection{Six dimensional effective field theory}

The six dimensional field theory that remains after compactification of the IIA supergravity and ignoring all massive Kaluza Klein modes can be consistently truncated to:

$$
S=\int \sqrt{-g}\left(R-(\partial \sigma)^{2}-e^{-\sigma} \operatorname{tr}|F|^{2}\right),
$$

where $\sigma$ is the $6 \mathrm{~d}$ dilaton and the trace is taken over the color indices of the $S U(2)$ gauge field. Monopole solutions from (2.7) are obtained with a spherically symmetric ansatz for the metric:

$$
d s^{2}=-e^{\lambda(r)} \Delta(r) d t^{2}+d r^{2} / \Delta(r)+r^{2} d \Omega_{4}^{2},
$$

in terms of functions $\lambda$ and $\Delta$, and $d \Omega_{4}^{2}$ which is the invariant metric over the 4-sphere, together with the Yang monopole field strength and radial ansatz $\sigma(r)$ for the dilaton.

The dilaton cannot be consistently set to a constant and then eliminated from the action. So the solutions which minimize the action (2.7) are in principle different from those of a pure Yang-Mills theory to which the Yang monopole belongs. However, because the Yang-Mills field strength 2-form for Yang monopoles solution has components only on the 4-sphere, it continues to minimize the action (2.7) and solve the Yang-Mills equations as modified by the dilaton. This is the reason why we still keep the name.

\subsection{Dual model in Type IIB superstring}

As we will see in the following, the appearance of the enhançon mechanism and the fuzzy geometry in the realization of the Yang monopole we describe in this paper is better visualized in a dual model on Type IIB side. In section 2.2 we related our D2-D4 system on K3 setup with the M-Theory (and heterotic string theory) model proposed in [7] by S-duality. As already noticed in [10], a configuration with $N \mathrm{D}(p+$ 
1)-branes stretched between two NS5-branes is T-dual to the same number of $\mathrm{D}(p+2)$ branes wrapped on the two-cycle of an $A_{1}$ ALE space, giving rise to $N$ effective $\mathrm{D} p$ branes. This means that the D2-D4 brane on a vanishing 2-cycle of $\mathrm{K} 3$ setup is dual to a D1-brane and a D3-brane stretched between two approaching NS5-branes, the vanishing limit of the IIA picture corresponding to the coalescence of the NS5-branes. The spatial position of the branes in the IIB picture can be described as follows. Let us state that both NS5-branes occupy dimensions 012345. The D1-brane extends along a transversal dimension (say, 6) and has an end on each NS5-brane. The D3-brane, which is actually supporting the magnetic charge of the configuration, shares the last direction plus two transversal dimensions more, which will be named 78. These two new directions correspond to the two remaining compact dimensions of $\mathrm{K} 3$ that not belong to the vanishing 2-cycle.

Note, that the D3-brane is effectively a D1-brane with an infinte mass, given that the two remaining directions, parallel to the NS5-brane, are infinite. So, in the limit where the two NS5 coincide, the D3-brane must be interpreted as an infinite D1-brane pulling at both NS5-branes. Thus, the D3-brane is seen as a point from the NS5-brane, in a way that the nontrivial magnetic configuration it carries is point-like in the (5+1)dimensional brane world. The fact that the effective D1-brane has infinite mass is the reason why the Yang Monopole in our brane picture has infinite energy, in agreement with the well-known gauge computation. The use of the other transverse D1-brane, which sizes goes to zero, is not other but to enhance the gauge symmetry.

\section{$3 S O(4)$-monopoles}

Another D-brane construction can be achieved if we consider a $S O(4)$ gauge group instead of $S U(2)$. This object will be called extended-Yang monopole [9]. The procedure is similar to the one shown in 2.1. Before entering into details let us first notice that the existence of 2 charges in the usual Yang construction is not obvious from the brane picture. D4-branes and D4-antibranes can each wrap in two different ways a 2-cycle. The number of charges would be in principle 4, each one labeling a possible realization. This is not the case as we are going to see. Let us call

$$
\lambda_{i}: S^{2} \longrightarrow S^{4} \quad i=1,2
$$

the two homotopically inequivalent maps of (2.3), corresponding to the two ways a D4-brane wraps a 2-sphere, and $\tilde{\lambda}_{i}$ the homologous maps for a D4-antibrane. The point is that there exists a homotopic deformation which makes $\lambda_{1}=\tilde{\lambda}_{2}$ and $\lambda_{2}=\tilde{\lambda}_{1}$, leaving us with just 2 (plus the trivial) homotopically inequivalent maps and, con- 

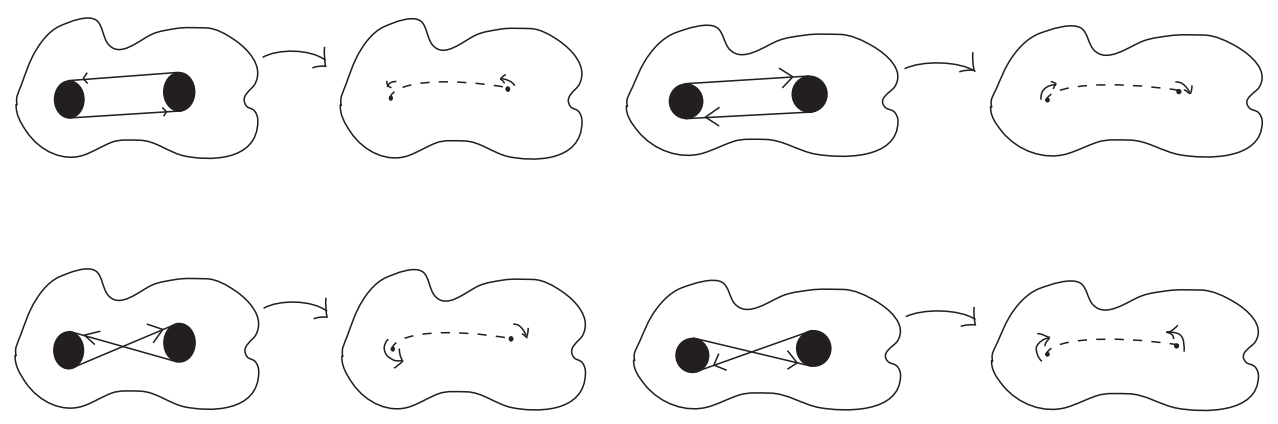

Figure 1: The four ways a D4-brane can wrap two distinguishable vanishing 2-cycles of the K3 surfcae. The configurations are homotopically different and so the number of charges is four. Notice that at the singular limit (right) the 2-dimensional part of the D4-brane connecting the two shrinking cycles vanishes into a string and just the two wrapped cycles remain.

sequently, 2 charges. Roughly speaking, this means that the "one-way" wrap of the D4-brane is actually identified with the "other-way" wrap of the D4-antibrane.

Things are different if we consider an extended-Yang monopole. As shown in [9], imposing spherical symmetry to a $S O(4)$ bundle over $R^{5}-0$ leaves four homotopically different possibilities. So the number of charges is 4 in this case. This is intimately related to the fact that the algebra of $S O(4)$ is isomorphic to the Cartesian product of two copies of $\mathfrak{s u}(2)$, as can be visualized in its Dynkin diagram. In the brane picture, the isomorphism of the algebras together with the geometric engineering mechanism we have used along subsection (2.1), suggests that the construction of the $S O(4)$-monopole involves two vanishing 2-cycles on K3. Recall that in the ALE space, each vanishing cycle is an $A_{1}$ singularity where the D2-brane is wrapped. Now, two D2-branes wrap a shrinking cycle each, and geometrically engineer a $\mathfrak{s u}(2)$ factor. The singularities are well separated 6 and disconnected. They are distinguishable. Now, as in the Yang case, we wrap them with a D4-brane (see figure), which also wraps the rest7 of the K3 surface. As before, the "one-way" wrap of the D4-brane is identified with the "other-way" wrap of the D4-antibrane, so only branes are considered. Now, the fact that $A_{1}$ singularities are distinguishable rises the number of possible inequivalent configurations to 4 . The homotopy group which labels the homotopically inequivalent maps is now

$$
\pi_{4}\left(A_{1} \times A_{1}\right)=\pi_{4}\left(A_{1}\right) \times \pi_{4}\left(A_{1}\right)=\mathbb{Z}_{2} \times \mathbb{Z}_{2},
$$

corresponding to the 4 charges for the extended-Yang monopole, in agreement with [9].

\footnotetext{
${ }^{6}$ Strictly speaking, they would actually be at an infinite distance in the ALE space.

${ }^{7}$ Think, for example, that K3 occupies dimensions 6789 . The 2-cycles may be both placed at 67 and the D4-brane wraps it all.
} 


\section{The multi-charge disease and the enhançon cure}

As mentioned in the introduction, there is an apparent inconsistency in the D2-D4 system on K3 model we have just considered and which we claim as the Yang monopole. It precisely concerns its charges. There is a simple mechanism which would allow in principle to add an infinite charge to the monopole without breaking the spherical symmetry. It would contradict the two charge nature of the Yang monopole and, consequently, the model would be incomplete. This is not the case though. We devote this section to this issue.

\subsection{The problem}

The D4-D2 brane model proposed in [1] seemed to fit the Yang monopole gauge solution [2] nicely: the dimension was correct; the $S U(2)$ gauge group was geometrically engineered by the D2-brane; the magnetic charge, carried by the D4-brane, had just two possible configurations in accordance with the the two charges the Yang solution permits; spherical symmetry was manifest by construction; the model happened to be dual to another [19] who claimed to have given a realization of the Yang monopole in M-theory... However, as compared to the well-studied gauge solutions, there was a crack in our brane model concerning the multiple charge configurations and the (apparently) unavoidable spherical symmetry the model showed. It has been objected that the model was not by itself capable to explain the two-charge quality of the Yang monopole as opposed to the Z-tower of charges that characterizes Dirac monopoles 8 . The objection takes into account that there is a simple mechanism which would allow in principle to add an infinite charge to the monopole without breaking spherical symmetry. It soon urged for a deeper study since it would contradict the two charge nature of the Yang monopole and, consequently, the model proposed in [1] would be incomplete.

Let us go a little deeper in the problem and explain how the model cures itself. Consider a D4-brane wrapped on the K3 surface and located at $r=0$ in a polar coordination of the six dimensional spacetime. Being BPS states there is no reason against the addition of a second, a third or subsequent D4-brane superpositions. It results in a pile of an arbitrary number of D4-branes wrapped on the K3 surface at the same location, say $r=0$. Each brane accounts for a unit of magnetic charge, so the total charge is basically the number of D4-branes which is, in principle, arbitrary. Besides, the configuration manifestly preserves spherical symmetry from the six dimensional spacetime point of view since all the branes are located at the same point. In essence,

\footnotetext{
${ }^{8}$ We thank P. K. Townsend for this remark.
} 
this simple mechanism allows us to construct a monopole with arbitrary charge from our model which, for this reason, could no longer be claimed as the Yang monopole. Is there any way out?

Before answering the question, let us analyze the argument a little deeper. In the original paper[2], Yang did not merely construct the field strengths for the monopoles but also, and by means of the construction itself, proved that (up to isomorphism) there are only two nontrivial static spherically symmetric bundles of $S U(2)$ over $R^{5,1}$ $\{0\}$. They are respectively labeled by $\{+1,-1\}$ charges?. Now, it seems inappropriate to claim the model as the Yang monopole when its internal logic (the brane superposition) allows to add arbitrary charge. The field configurations they correspond to are either not $S U(2)$-bundles or they are simply not spherically symmetric. However, both properties have been explicitly and intentionally imposed in the construction of our brane model. That is the problem.

The enhançon mechanism (or more precisely, the fuzzy geometry it involves for multicharge configurations), by means of which the second and subsequent branes feel a repulsive interaction with respect to the first one and cannot reach the origin but smear onto the enhançon locus 10 at radius $r_{e}$, comes as a surprising solution for our problem. It is also a non trivial prove of consistency of the brane model we propose for the Yang monopole, a pure stringy mechanism that comes for the brane picture to agree with a purely gauge theory requirement. The enhançon mechanism was originally found in $\mathrm{D} p / \mathrm{D}(p+4)$-branes wrapped in regular $\mathrm{K} 3$ surface [10], bu it also works for fractional branes [13].

\subsection{The enhançon}

In the picture shown in section 2 of the D2-D4 system on K3 model we did not make any assumption about the energy of the brane states. It was not necessary since our discussion was purely geometrical and did not need the description of an explicit six dimensional effective field theory. In the following discussion however it becomes essential for the branes to be BPS states.

When originally proposed[10], the authors realized that their new mechanism (the enhançon) could resolve a naked timelike singularity produced by a $\mathrm{D} p$-brane wrapped in a 2-cycle of the $\mathrm{K} 3$ surface which was being called repulson 11 . In order to

\footnotetext{
${ }^{9}$ A systematic analysis of the possible static spherically symmetric $S O(2 n)$-bundles over $R^{2 n+1,1}$ $\{0\}$, and consequently, of the charges that label them, is carried out in[9].

${ }^{10}$ The enhançon is, by definition, the locus of points where a probe brane gets tensionless in certain brane configurations as it tries to reach the origin.

${ }^{11}$ In the geometry of a repulson, a massive particle would naively feel a repulsive gravitational force
} 
investigate it, they used the 10-dimensional supergravity of the system D2-D6 on K3 model although they showed that the same conclusions hold for any $\mathrm{D} p-\mathrm{D}(p+4)$ on K3 mode 12. The geometric locus of the enhançon is independent of the model. Let us see how the enhançon comes up in our model, for the case $p=0$.

We will use the D0/D4-branes on the K3 surface, that is, without including D2branes, so the gauge theory is abelian in the low energy approximation that follows. D2-branes are not relevant for the phenomenon we are describing, they do not "see" the enhançon shell. This fact allows us to simplify the computation but still trust the result as extended for the full model.

The simplest static supergravity solution consistently truncated to its bosonic part can be written as:

$$
\begin{aligned}
d s^{2} & =-Z_{0}^{-1 / 2} Z_{4}^{-1 / 2} d t^{2}+Z_{0}^{1 / 2} Z_{4}^{1 / 2}\left(d r^{2}+r^{2} d \Omega_{4}^{2}\right)+V^{1 / 2} Z_{0}^{1 / 2} Z_{4}^{-1 / 2} d s_{K 3}^{2}, \\
e^{2 \Phi} & =g_{s} Z_{0}^{3 / 2} Z_{4}^{-1 / 2} \\
C_{(1)} & =\left(Z_{0} g_{s}\right)^{-1} d t \\
C_{(5)} & =\left(Z_{4} g_{s}\right)^{-1} d t \wedge \epsilon_{K 3} .
\end{aligned}
$$

The line element corresponds to the string frame. $d S_{K 3}^{2}$ is the metric of the K3 surface of unit volume, and $\epsilon_{K 3}$ is its corresponding volume form. Providing that the solution is asymptotically flat, the harmonic functions are:

$$
\begin{aligned}
& Z_{0}=1-\frac{V_{*}}{V} f(r), \\
& Z_{4}=1+f(r),
\end{aligned}
$$

where $V$ is the volume of K3 at $r=\infty$ and $V_{*}=\left(4 \pi l_{s}\right)^{4}=\frac{\mu_{0}}{\mu_{4}}$. The volume of K3 at arbitrary $r$ can be read off from (4.1):

$$
V(r)=V \frac{Z_{0}(r)}{Z_{4}(r)}
$$

And the fuction $f(r)$ is, for a solution with $N$ branes:

$$
f(r)=\frac{1}{4} \frac{N g_{s} l_{s}^{3}}{r^{3}}=\frac{V}{V_{*}} \frac{r_{0}^{3}}{r^{3}}
$$

where $r_{0}$ is the radius where a naked singularity (repulson) is placed, as confirmed by inserting (4.4) in (4.1) and computing the Kretschmann curvature scalar. As argued

by a potential which becomes infinite as the particle approaches a point at finite distance from the physical location of the branes.

${ }^{12} \mathrm{D} p$-branes are in the solution for consistency since even if one does not put them by hand they virtually appear. 
in [10], this singularity is unphysical. This may be seen by probing the geometry (4.1) with other D4-brane. The action of the probe may be written as

$$
S_{\text {probe }}=\int d t e^{-\Phi} \underbrace{\left(\mu_{4} V(r)-\mu_{0}\right)}_{m(r)} \sqrt{-g}+\mu_{4} \int C_{5}-\mu_{0} \int C_{1},
$$

where the function $m(r)$ of the DBI term is the mass of the probe. The probe becomes massless at $m(r)=0$, that is when $V(r)=V_{*}$. We will define $r_{e}$ as the point where the $\mathrm{K} 3$ volume becomes $V_{*}$, so $V\left(r_{e}\right)=V_{*}$. Now, $r_{e}$ defines the enhançon locus. Its value can be easily computed:

$$
f(r)=\frac{1}{2}\left(\frac{V}{V_{*}}-1\right) \longrightarrow r_{e}=\frac{2 V}{V-V_{*}}\left|r_{0}\right|>\left|r_{0}\right| .
$$

The last inequality of (4.6) shows that the enhançon radius is always bigger than $r_{0}$, the radius where the naked singularity is placed, provided that $V>V * 13$.

For smaller values of $r$ than the enhançon radius the tension of the probe becomes negative and the solution is considered unphysical from the supergravity perspective. The probe cannot approach the point $r=0$. Moreover, as "seen" by the probe, the $N$ D4-branes are not at $r=0$ but smeared over the enhançon locus as well.

If we now try to start building the model, say, from empty space, the first D4-brane does not present any enhançon difficulties to reach $r=0$, so the 1-(anti)brane solution is spherically symmetric. However, as we try to add a second and subsequent branes to the former, the enhançon mechanism prevents them to reach the first brane at the origin. Even if its not straightforward to see that this breaks spherical symmetry (the enhançon shell is, in principle, a sphere), it is not hard to believe that lack of point-like behavior will trigger multipole contributions. We will devote the next subsection to debate on this issue. For the time being let us accept it, and prelude that the model itself dynamically cures its apparent multi-charge contradiction.

There are two remarks that are worth pointed out. First, it should be noticed that the branes considered in seminal papers [10, 11, 12] were wrapping a nonvanishing 2-cycle of the K3 surface. Our model, by contrast, is built by the so-called fractional branes, that is, $\mathrm{D} p$-branes which wrap vanishing 2-cycles of the K3 surface 14 . This difference could raise some doubts on the above arguments. However, fractional brane solutions have also been proved to show enhançon behaviour[13]. A second remark concerns about the gauge symmetry. As said before, although it is true that the explicit calculations were carried out only for an abelian gauge field, the extension for a

\footnotetext{
${ }^{13}$ The supergravity equations imply that $V(r)$ is an increasing function. So $V=V(r \rightarrow \infty)>V_{*}$ is always true.

${ }^{14} \mathrm{D} p$-branes on the $\mathrm{K} 3$ surface are equivalent to $\mathrm{D}(p-2)$-branes on $T^{4} / Z_{2}$, the orbifold limit of the K3 surface. So D4-branes becomes D2, and D2 becomes D0 fractional branes.
} 
$S U(2)$ Yang-Mills field (as geometrically engineered in our model) is not expected to show any obstruction for the enhançon given that the D2-brane is not affected by the enhançon.

\subsection{A way out of spherical symmetry}

As said some lines above is is not the enhançon mechanism but the fuzzy geometry it involves what is behind the whole reasoning. Let us see that in detail.

In their first papers[10, 11], the authors considered the enhançon locus as the spherical shell of radius $r_{e}$ where the stuck of $N$ branes become massless and smeared out homogenously. So, even when the system itself prevents the branes from piling at a six-dimensional point, say $r=0$, it seems that the solution is still isotropic and then spherically symmetric. The original picture of the enhançon does not actually break the spherical symmetry of the $N>1$-configuration of wrapped D4-branes as claimed above.

It happens, however, that the homogenous distribution of $N$ branes over the spherical enhançon shell is, as noticed in [14, 26], just a particular supergravity solution. The enhançon, in general, has arbitrary shape. Think for example of an oblate shape. One can then define a brane density on this enhançon surface whose integral gives back the $N$ constituent branes. This density is not homogenous. Moreover, as ruled by supergravity, for static solutions the brane density on the enhançon behaves very much like an electrostatic distribution of charges on a metal [14], that is, growing in regions where the curvature of the enhançon surface is higher.

The spherical shape of the enhançon shell is then a particular case of a broad family. The sphere was originally encountered not only for simplicity, but also because the geometry was tested with a probe. Let us explain this point. The usual procedure for the study of the enhançon geometry has been to take an initial set of $N$ gravitating branes located at the origin. They are consequently a source of a spherically symmetric geometry of the supergravity solution. Now, take the probe to test it. As the back reaction of the probe is (by the definition of probe) not taken into account, there is in principle no natural mechanism in the setup that might break spherical symmetry. So, what was the point in considering different-from-spherical enhançon shells?

Consider the case of two monopoles. The first brane is placed at the origin so it is one monopole. Now try to bring another real brane to wrap on it, another brane on the same footing not a probe. The six dimensional supergravity ansatz for the metric of this brane setup is clearly axially symmetric. So, what is the point in considering that the branes finally melt out in a spherical enhançon shell? 
The above argument suggests that there is in principle no especial preference for spherical shells. Moreover, that a non spherical geometry for the enhançon locus is naturally favoured. However, although suggesting, the argument is inconclusive. Sphericalness may seem capricious but still possible within the brane picture whereas it is forbidden in a two or higher charge configuration from the field theory point of view. A satisfactory dynamical mechanism should rule out spherical enhançon shells.

Fortunately, there is an elegant way out the spherically symmetric puzzle which involves phenomena beyond SUGRA. Recall that the enhançon locus is the place where the probe brane (and also the gravitating one as seen by the probe) becomes tensionless. At large distance from $r=0$ the probe was pointlike in the six noncompact dimensions. However as the distance to the center approaches $r_{e}$ the brane seems to "emerge" and smear on a four dimensional sphere over the noncompact dimensions. Some lines above, it was explained that the geometry of the enhançon need not to be spherical but a large family of shapes are both available an consistent with the enhançon mechanism [14, 26]. This was a comforting way out of the necessity of spherical symmetry but left us with an uneasy family of arbitrary shapes which are all on equal footing with the sphere. Why are all valid? Is there any mechanism that rules out any of them? It seems a little naive though to think of such sharp geometries at a region where the branes are blown in new (noncompact) dimensions and spacetime seem not to behave ordinarily. Indeed, it is believed that the correct description of the geometry near the enhançon locus is a fuzzy sphere [27]. Fuzzy spheres and more generally, non-commutative geometries break unavoidably spherical symmetry. Moreover, Riemannian geometry and the concept of manifold are no longer valid in this context. It is remarkable, besides, that the fuzzy geometry appears for $N>1$ and so the charge- 1 monopole does not get affected and recovers spherical symmetry as expected. We will indicate within the next sections the relation between the brane setup, the monopoles and the fuzzy geometry.

\section{Connection with fuzzy spheres}

Fuzzy spheres are examples of noncommutative geometry [27]. The main idea that underlies the construction of these spaces is the one-to-one correspondence between the differential geometry of manifolds and the commutative algebra of functions defined on them. The coordinates are the generators of the algebra and the vector fields are the derivations. A fuzzy sphere differs from an ordinary sphere because the algebra of functions on it is not commutative. Taking spherical harmonics as a basis, fuzzy spheres are generated by harmonics whose spin $l$ is not greater than a given 
$j$. The terms in the product of two spherical harmonics that involves harmonics with exceeding $j$ are just omitted. This truncation makes the algebra of functions noncommutative. So fuzzy spheres are labeled by an integer number $j$. For $j=2$, the algebra describes poorly a $S^{2}$-sphere, in fact, only the north and the south pole are distinguishable. For $j=3$ one can make out the equator as well, so the geometry gets less fuzzy. The ordinary sphere is recovered in the limit $j \rightarrow \infty$. In what follows we will turn $j$ into $N$, the number of branes, because as we will see, the number of branes will actually rule the degree of fuzziness. The radius of the fuzzy sphere is given by

$$
R^{2}=\kappa\left(N^{2}-1\right)
$$

where $\kappa$ must be proportional to $\alpha^{\prime}$ for dimensions.

In which sense are these fuzzy geometries related to the multiple charge configurations? Unfortunately, we do not know the proper mathematical framework to show how fuzzy geometry comes up in the world volume of the NS5-branes in our model, as nicely shown in [17] (by means of exploiting the DBI action) for non abelian and non BPS setups where a pile of $N$ D1-branes stretch between two D5-branes. We lack a low energy action for the worldvolume of NS5-branes. However, it is commonly believed that the enhançon mechanism comes along with fuzzy geometry, and in the case of fuzzy spheres, (5.1) holds for the fuzzy radius, where $N$ is the number of transversal branes. It is clear from (5.1) that $N=1$ do not lead to fuzziness, but for any $N>1$ it does. So multicharge solutions are fuzzy.

There is a remarkably nice explanation of how fuzzy geometry comes out from a similar setup [10] to our model. It is worth reviewing it in this section.

The model they used was built with D6-D2 brane system wrapped on the K3 surface. In previous works [11], the authors already noticed that such system would make a t' Hooft-Polyakov monopole when dimensionally reduced to four dimensions. Specifically, they consider a D6-brane wrapping the K3 surface and two more compact flat dimensions, where the D2-brane lives. The enhançon mechanism produces an enhancement of the gauge symmetry $U(1) \rightarrow S U(2)$ in the 4-dimensional region $r<r_{e}$, which will be the core of the monopole. The configuration gets Higgsed as one moves far from $r_{e}$ recovering the $U(1)$-magnetic charge.

In order to better visualize it, the brane setup that was actually used [15, 16] to show how fuzzy spacetime geometry enters in the enhançon picture was not the D6D2 system on K3 model already mentioned but a dual one in Type IIB instead, which consists of a pair of parallel NS5-branes with $N$ D3-branes stretched between them. The separation of the NS5-branes is parameterized by $\sigma \in[-1,+1]$ at which extremes the branes are initially located. The presence of the D3-branes deforms the geometry 
of the NS5-branes into a double trumpet shape [15]. The enhcançon locus is precisely a transversal section of the bunch of D3-branes at the point (say $\sigma=0$ ) where the two NS5-branes make contact. As a consequence of the two brane connection, the gauge symmetry gets enhanced to $\mathrm{SU}(2)$ at the enhançon locus. That was expected. It is also expected that $N$ BPS monopoles enter this picture. They are placed at the ends of the D3-branes as seen by the part of the NS5-brane worldvolume transverse to the D3-brane. The positions of those ends can be coordinated by $\Phi^{i},(i=1,2,3)$. This "coordinates" fulfill Nahm equations in the BPS case:

$$
\frac{d \Phi^{i}}{d \sigma}=\frac{1}{2} \epsilon_{i j k}\left[\Phi^{j}, \Phi^{k}\right] .
$$

The appropriate solutions (BPS monopoles) are those for which the $\Phi^{i}(\sigma)$ have a single pole at the end of the interval $\sigma \in[-1,+1]$ and the residues $\Sigma^{i}$ form the $N \times N$ irreducible representation of $S U(2):\left[\Sigma^{i}, \Sigma^{j}\right]=2 i \epsilon_{i j k} \Sigma^{k}$. The general solution of this kind takes one $S U(2)$ representation and twists it to another as it crosses the interval. The enhançon (recall, a section on the stuck of D3-branes) is a fuzzy sphere for finite $N \neq 1$ [27]. For example, for a two D3-brane setup, a section will capture only two clear points of $S^{2}$, the north and south pole. For a single D3-brane, however, the radius of the fuzzy sphere vanishes (5.1). For large $N$ the geometry becomes less fuzzy, recovering the usual $S^{2}$ as $N \rightarrow \infty$.

Their construction is different from the D4-D2 system on the K3 surface we propose for the six dimensional Yang monopole. Differences include, for instance, the origin of the enhanced $S U(2)$ symmetry. We did not make use of the enhançon mechanism but we geometrically engineered the gauge group instead, as explained in section 2. We did so in order to account for a $S U(2)$ gauge group all over the six dimensional space (and not only within the enhançon region), as needed for the Yang monopole configuration. Unfortunately, for the case D1-branes stretched between a pair NS5-brane (our T-dual model), we lack the Nahm equations and an analogous procedure as just shown for the emergence of fuzzy spheres cannot be set. It would be very interesting to study the analogous algebra in our model for the emergence of the fuzzy 4-sphere at the enhançon locus. We leave it for a future work.

Despite this and other obvious differences the essence of the problem concerning spherical symmetry remains. It is because the enhançon shell they considered was spherical and supported homogenously a melting $N$ D6-branes on it. An inconsistency with field theory is again encountered (and cured by the fuzzy geometry) in their case. That is because spherically symmetric $t^{\prime}$ Hooft-Polyakov monopoles with multiple magnetic charge do not exist [28].

Needles to say that the same enhançon-fuzzy mechanism applies for the $S O(4)$ 
monopole and cures it from the multicharge disease which could undergo in the brane picture had the geometry not become fuzzy.

\section{Discussion and open questions}

In this paper, a Type IIA geometric realization of the Yang monopole in six dimensions given in [1] is revisited and its apparent contradictions are clarified. In the construction of the magnetic object, it has been used the result of the duality between Type IIA superstring compactified on the K3 surface and heterotic superstring on $T^{4}$. The $S U(2)$ gauge symmetry of the Yang monopole is considered as the enhanced gauge symmetry corresponding to shrinking 2-cycles inside the K3 surface, and the Yang monopole comes up by wrapping D-branes on the K3 non-trivial cycles. In this way, the properties of the Yang monopole are encoded in the K3 surface data.

With respect to the charges of the configuration, suggestions and objections that came up during the presentation of [1] have been taking in full consideration. In our opinion, the present work brings light to the main objections strengthening and completing the brane picture of the Yang monopole. Firstly, it was claimed that the number of charges of one D4-brane setup should be four, as accounted for the two ways the brane and the antibrane can wrap a 2-cycle. The answer is given at the end of section 2, There it is explained that the four configurations are actually identified in pairs, so it results in just two homotopically different configurations. Indeed, as explain in the same section, the $S O(4)$ extended-Yang monopole is the one who carries four charges and a brane picture for it is proposed.

The second objection have been taken into analysis in section 4. When more than one D4-brane are added to the model, its interpretation as a Yang monopole gets into trouble since and infinite tower of charges seem to appear. This is what we have called multicharge disease. The multi-charge problem of this construction gets satisfactory solved by the dynamics of the enhançon mechanism which, as explained in section 4, ruins spherical symmetry in the multi-brane setup and then saves the model from contradiction.

The question of how such an involved concept as the enhançon locus and its correlated fuzzy geometry comes into stage to make the brane configuration non spherically symmetric as required, for different reasons, by the gauge field theory is a point that in our opinion requires further analysis. More on this will be reported in future works. 


\section{Acknowledgements}

We thank M. Asorey, L. J. Boya, E. H. Saidi, P. K. Townsend, K. Goldstein and C. Hoyos-Badajoz for discussions and enlightening comments. This work has been partially supported by CICYT (grant FPA-2009-09638) and DGIID-DGA (grant 2007-E24/2). We thank also the support by grant A9335/07 and A9335/10 (Fisica de alta energia: Particulas, cuerdas y cosmologia).

\section{References}

[1] A. Belhaj, P. Diaz, A. Segui, On the Superstring Realization of the Yang Monopole (2007), hep-th/0703255.

[2] C. N. Yang, Generalization of Dirac's Monopole to $S U_{2}$ Gauge Fields, J. M. Phys. 19 (1978)1.

[3] P.A.M. Dirac, Quantized Singularities in the Electromagnetic Field, Proc. Royal Soc. London, A133 (1931) 60.

[4] A. A. Belavin, A. M. Polyakov, A. S. Shvarts and Yu. S. Tyupkin, Pseudoparticle Solutions of the Yang-Mills Equations, Phys. Lett. B 59, (1975) 85.

[5] Z. Horvath, L. Palla, Spontaneous compactification and monopoles in higher dimensions, Nucl. Phys. B142 (1978) 327.

[6] T. Tchrakian, Dirac-Yang monopoles and their regular counterparts, hep-th/0612249.

[7] G. W. Gibbons, P. K. Townsend, Self-gravitating Yang Monopoles in all Dimensions, Class. Quant. Grav. 23 (2006) 4873, hep-th/0604024.

[8] P. Diaz and A. Segui, Generalized Nariai Solutions for Yang-type Monopoles, Phys. Rev. D 76 (2007) 064033, arXiv:0704.0366 [gr-qc] .

[9] P. Diaz, A. Lazaro, Monopoles in Arbitrary dimension, arXiv:0811.4187.

[10] C. V. Johnson, A. W. Peet and J. Polchinski, Gauge Theory and the Excision of Repulson Singularities, Phys. Rev.D61 (2000) 086001, hep-th/9911161.

[11] C. V. Johnson, R. C. Myers, A. W. Peet and S. F. Ross, The enhançon and the Consistency of Excision, Phys. Rev. D64 (2001) 106001, hep-th/0105077. 
[12] A. Dimitriadis, A. W. Peet, G. Potvin and S. F. Ross, Enhanonn Solutions: Pushing Supergravity to its Limits (2003), hep-th/0311271.

[13] M. Frau, A. Licardo and R. Musto, The Geometry of Fractional Branes (2000), hep-th/0012035.

[14] D. Astefanesei and R. C. Myers, A new wrinkle on the enhançon (2001), hep-th/0112133.

[15] C. V. Johnson, Enhançons, Fuzzy Spheres and Multi-Monopoles, hep-th/0004068.

[16] C. V. Johnson,The enhançon, Multimonopoles and Fuzzy Geometry, hep-th/0011008.

[17] N.R. Constable, R.C. Myers, O. Tajford,Non-abelian Brane Intersections, JHEP 0106 (2001) 023, hep-th/0102080.

[18] J. Polchinski, Open Heterotic Strings, JHEP 0609 (2006) 082, hep-th/ 051003.

[19] E. A. Bergshoeff, G. W. Gibbons, P. K. Townsend, Open M5-branes, Phys. Rev. Lett. 97 (2006) 231601, hep-th/0607193.

[20] B. Chen, H. Itoyama, H. Kihara, Non Abelian monopoles from matrices, Nucl. Phys. B577 (2000) 23, hep-th/9909075.

[21] E. Witten, Phases of $N=2$ Theories in Two Dimensions, Nucl. Phys. B 403 (1993) 159, hep-th/9301042.

[22] S. Katz, A. Klemm, C. Vafa, Geometric Engineering of Quantum Field Theories, Nucl. Phys. B497 (1997) 173, hep-th/9609239.

[23] B. Acharya, E. Witten, Chiral Fermions from Manifolds of $G_{2}$ Holonomy, hep-th/0109152.

[24] A. Belhaj, Manifolds of $G_{2}$ Holonomy from N=4 Sigma Model, J. Phys. A35 (2002) 8903, hep-th/0201155.

[25] C. Vafa, Lectures on Strings and Dualities, hep-th/9702201.

[26] L. M. Dyson, L. Jarv, C. V. Johnson, Oblate, Toroidal, and Other Shapes for the Enhançon, hep-th/0112132.

[27] J. Madore, The fuzzy sphere, Class. Quantum Grav. 9 (1992)69.

[28] E. J. Weinberg and A.H. Guth, Nonexistence of spherically symmetric monopoles with multiple magnetic charge, Phys. Rev. D14 (1976) 1660. 